\title{
Study on phase shifted fiber Bragg grating spatial sensing properties to ultrasonic wave at arbitrary excitation angle
}

\author{
Meng Lijun ${ }^{1 \mathrm{a})}$, Yi Jiangang ${ }^{1}$, Tan $\mathrm{Xin}^{1}$, and $\mathrm{Cai} \mathbf{L i}^{2}$ \\ ${ }^{1}$ School of Electromechanical and Architectural Engineering, Jiang han University, \\ No. 8 Delta Lake Road, Wuhan, Hubei, China \\ ${ }^{2}$ School of Mechanical and Electronic Engineering, Wuhan University \\ of Technology, \\ No. 122 Luoshi Road Wuhan, Hubei, China \\ a)menglijun0408@163.com
}

\begin{abstract}
Taking into account the birefringence effect, the sensing models of phase shifted fiber Bragg grating (PS-FBG) under ultrasonic wave from arbitrary excitation angle were established, and its reflection spectrums were analyzed by simulation based on the transfer matrix principle. Under the excitation of transverse ultrasound, the PS-FBG sharp dip was split into two peaks due to birefringence, the split point spacing increased with the transverse force; under the effect of the ultrasonic wave from random angle, the PS-FBG signal intensity and the ultrasonic excitation angle presented a Cosine curve with two peaks in the middle. Finally, the relevant conclusions were verified by experiments and $12.11 \mathrm{MHz}$ ultrasonic wave was obtained by PS-FBG.
\end{abstract}

Keywords: phase shifted fiber Bragg grating, ultrasonic wave, spatial sensing properties, excitation angle

Classification: Optical systems

\section{References}

[1] C. Li, et al:: "Application of interpolation algorithm in plate structure detection based on fiber Bragg grating sensing technology," IEICE Electron. Express 12 (2015) 20150271 (DOI: 10.1587/elex.12.20150271).

[2] J.-R. Lee, et al.: "Impact wave and damage detections using a strain-free fibre Bragg grating ultrasonic receiver," NDT\&E Int. 40 (2007) 85 (DOI: 10.1016/ j.ndteint.2006.07.001).

[3] H. Tsuda, et al:: "Fatigue crack propagation monitoring of stainless steel using fiber Bragg grating ultrasound sensors," Smart Mater. Struct. 15 (2006) 1429 (DOI: 10.1088/0964-1726/15/5/032).

[4] N. Takeda, et al.: "Development of smart composite structure with smalldiameter fiber Brag grating sensor for damage detection: quantitative evaluation of delamination length in CFRP laminates using Lamb wave sensing," Compos. Sci. Technol. 65 (2005) 2575 (DOI: 10.1016/j.compscitech. 2005.07.014). 
[5] Y. Qian and S. C. Yu: "Transmission spectrum of the shifted fibre bragg grating," J. China Univ. Metrol. 20 (2009) 324.

[6] Q. Wu and Y. Okabe: "High-sensitivity ultrasonic phase-shifted fiber Bragg grating balanced sensing system," Opt. Express 20 (2012) 28353 (DOI: 10. 1364/OE.20.028353).

[7] Q. Wu, et al.: "Sensitivity distribution properties of a phase-shifted fiber Bragg grating sensor to ultrasonic waves," Sensors 14 (2014) 1094 (DOI: 10.3390/ s140101094).

[8] Q. Wu and Y. Okabe: "Novel real time acousto ultrasonic sensors using two phase-shifted fiber Bragg gratings," J. Intell. Mater. Syst. Struct. 25 (2014) 640 (DOI: 10.1177/1045389X13483028).

[9] T. Fink, et al:: "Study of $\pi$-phase-shifted fiber-Bragg-gratings for ultrasonic detection," Proc. SPIE 8370 (2012) 83700M (DOI: 10.1117/12.920810).

[10] A. Rosenthal, et al.: "High-sensitivity compact ultrasonic detector based on a pi-phase-shifted fiber Bragg grating,” Opt. Lett. 36 (2011) 1833 (DOI: 10.1364/ OL.36.001833).

[11] T. Liu and M. Han: "Analysis of $\pi$-phase-shifted fiber Bragg gratings for ultrasonic detection," IEEE Sensors J. 12 (2012) 2368 (DOI: 10.1109/JSEN. 2012.2189383).

[12] J. Guo, et al.: "Ultrasonic imaging of seismic physical models using a phaseshifted fiber Bragg grating," Opt. Express 22 (2014) 19573 (DOI: 10.1364/OE. 22.019573).

[13] T. Erdogan: "Fiber grating spectra," J. Lightwave Technol. 15 (1997) 1277 (DOI: 10.1109/50.618322).

[14] P.-M. Lam, et al.: "Acousto-ultrasonic sensing for delaminated GFRP composites using an embedded FBG sensor," Opt. Lasers Eng. 47 (2009) 1049 (DOI: 10.1016/j.optlaseng.2009.01.010).

[15] A. B. Lobo Ribeiro, et al.: "Analysis of the reflective-matched fiber Bragg grating sensing interrogation scheme," Appl. Opt. 36 (1997) 934 (DOI: 10. 1364/AO.36.000934).

[16] R. Gafsi and M. A. El-Sherif: "Analysis of induced-birefringence effects on fiber Bragg gratings,” Opt. Fiber Technol. 6 (2000) 299 (DOI: 10.1006/ofte. 2000.0333).

[17] F. Kui, et al:: "Analysis of magnetic field sensor with phase-shifted grating based on the polarization properties," Chin. J. Lasers 37 (2010) 763 (DOI: 10. 3788/CJL20103703.0763).

\section{Introduction}

Ultrasonic excitation and fiber Bragg gratings (FBG) sensing technology possesses the FBG advantages such as resistance to electromagnetic interference, multi parameter distributed measurement, as well as the characteristics of ultrasonic detection, for instance strong penetration and good directivity. Its basic principle is to excite the mechanical structure by ultrasonic wave and measure the ultrasonic strain with FBG, then analyze the damage state of the mechanical structure [1]. However, in order to avoid the micro bending modulation of ultrasonic strain wave on FBG, the length of the FBG should be less than half of the ultrasonic wave length [2], therefore a shorter FBG is hoped; On the other hand, the center wavelength demodulation is based on optical power detection in ultrasonic excitation-FBG detection technology, namely using the narrow band optical filter to 
capture FBG spectrum under broadband light source or using laser source to intercept the FBG spectrum directly, and achieving the wavelength change by measuring the variation of the output light intensity [3], and when the light source intensity is certain, the demodulation sensitivity depends on the slope of the FBG spectrum, therefore it requires that the FBG length is as long as possible; in addition, ultrasound with high frequency (such as $\mathrm{MHz}$ ) is generally chosen in order to improve the damage detection accuracy, and the corresponding strain amplitude is very small (several or dozens of micro-strain) [4], therefore conventional FBG can not measure high-frequency ultrasound effectively. PS-FBG is a special fiber grating with discontinuous refractive index distribution, and it introduces a certain phase shift to one or more positions of the conventional FBG, opening one or more transmission windows with a narrow band width in the reflection spectrum [5]. Because of high wavelength selectivity and reflectivity as well as narrow line width, PS-FBG can be a preferred component for ultrasonic measurement in place of FBG.

At present, the research on ultrasonic excitation and PS-FBG distributed sensing damage detection technology is just getting started. Qi Wu et al. analyzed the influence of the distance and angles between PS-FBG and the ultrasonic source on the signal characteristic parameters $[6,7,8] . \pi$ PS-FBG was utilized for the acoustic emission detection of breaking lead by Thomas Fink et al. [9]. Rosenthal et al. studied on the feasibility of ultrasonic measurement using PS-FBG instead of FBG by experiment [10]. Tongqing Liu analyzed effect of the PS-FBG parameters for example grating length, grating refractive index modification depth on the its sensitivity for ultrasonic waves, and they believed that $\pi$ PS-FBG had similar directivity characteristics as Fabry-Perot cavities [11]. Jingjing Guo et al. carried out ultrasonic imaging study for the geophysical model with $\pi$ PS-FBG [12]. At present, the impact of the birefringence induced by lateral force on the PS-FBG response characteristics is not taken into account. In this paper, PS-FBG sensing models under ultrasonic waves from different directions were set up considering the birefringence effect, and PS-FBG reflection spectrums were studied based on the transfer matrix method; simulation and experiment were carried out to prove that the PS-FBG signal intensity and ultrasonic excitation angle present a Cosine relationship accompanied by two peaks in the central curve and PS-FBG can be used for the measurement of more than $10 \mathrm{MHz}$ ultrasound.

\section{Simulation model}

Based on the coupled mode theory and transfer matrix method [13], the PS-FBG is divided into $N$ segments of uniform fiber Bragg grating, and the transmission characteristic of each segment is characterized by a transmission matrix, therefore the whole transfer matrix of the PS-FBG will be got by multiplying each transmission matrix. In this paper, the main parameters of the $\pi$ PS-FBG were: effective refractive index $n_{\text {eff }}=1.45$, average refractive index change $\Delta n=1 \mathrm{e}-4$; fiber grating length $L=10 \mathrm{~mm}$; the phase shift is located in the middle of the grating region, the center wavelength $\lambda_{\mathrm{D}}=1550.05 \mathrm{~nm}$; elastic modulus $E=6.9 \mathrm{GPa}$ and Poisson ratio $v=0.16 ; P_{11}=0.121 ; P_{12}=0.27$. 


\section{Sensing model under axial ultrasonic wave}

To simplify the analysis, it is supposed that the ultrasonic wave is a cosine stress wave along the grating axis, and $\sigma_{\mathrm{x}}=\sigma_{\mathrm{y}}=0$ :

$$
\sigma_{z}=P(z, t)=P_{0} \cos \left(\omega t-\frac{2 \pi}{\lambda_{m}} z\right)=P_{0} \cos \left[2 \pi f\left(t-z / v_{m}\right)\right]
$$

$v_{\mathrm{m}}$ is ultrasonic velocity, and $P_{0}$ is ultrasonic stress amplitude. The PS-FBG fit the same wavelength change expression under the axial stress as FBG:

$\Delta \lambda_{x(y)}=-\frac{n_{e f f}^{3} \Lambda}{E}\left\{\left[\left(p_{11}-2 v p_{12}\right) \sigma_{x(y)}\right]+\left[(1-v) p_{12}-v p_{11}\right]\left(\sigma_{y(x)}+\sigma_{z}\right)\right\}+2 \frac{n_{e f f} \Lambda}{E}\left[\sigma_{z}-v\left(\sigma_{x}+\sigma_{y}\right)\right]$

As $\sigma_{\mathrm{x}}=\sigma_{\mathrm{y}}=0$, Formula (2) changes into

$$
\Delta \lambda=-\frac{n_{e f f}^{3} \Lambda}{E}\left\{\left[(1-v) p_{12}-v p_{11}\right] \sigma_{z}\right\}+2 \frac{n_{e f f} \Lambda}{E} \sigma_{z}
$$

The response sensitivity of PS-FBG to ultrasonic stress amplitude $P_{0}$ is:

$$
\frac{\Delta \lambda}{P_{0}}=\left\{1-\frac{n_{e f f}^{2}}{2}\left[p_{12}-v\left(p_{11}+p_{12}\right)\right]\right\} \frac{\lambda}{E}
$$

According to the simulation parameters, the response sensitivity of $\pi$ PS-FBG wavelength $\lambda$ to $P_{0}$ is $16.834 \mathrm{pm} / \mathrm{MPa}$. The strain in a test material induced by an ultrasonic wave is usually about several or over a ten micro strain. Suppose the test material is aluminum with $E=72 \mathrm{GPa}$, the maximum strain variation is $20 \mu \varepsilon$, therefore the corresponding $P_{0}=0.072-1.44 \mathrm{MPa}, \pi \mathrm{PS}-\mathrm{FBG}$ wavelength change is about $1.26-25.3 \mathrm{pm}$. The full width at half maxima (FWHM) of $\pi$ PS-FBG is usually about $30-50 \mathrm{pm}$. The PS-FBG measurement range is usually controlled within $20 \%-80 \%$ of its single slope in the demodulation system [14], so the $\pi$ PS-FBG measurement range is $6-40 \mathrm{pm}>1.26-25.3 \mathrm{pm}$. Under axial ultrasonic wave, PSFBG and FBG have the same wavelength sensing properties, and $\pi$ PS-FBG wavelength variation can be used for axial ultrasound measurement theoretically.

\subsection{Measurement sensitivity}

Fig. 1 displays the PS-FBG edge filter demodulation system based on tunable laser: a tunable laser is used to intercept the sharp dip of the PS-FBG reflection spectrum, and the photoelectric detector is employed to measure the light intensity change. The relationship between ultrasonic strain $\varepsilon$ and the output voltage $U$ of the edge filter demodulation system can be get based on Lobo Ribeiro's method [15]:

$U=K_{1} A I_{0} R_{s 0} \frac{\sqrt{\pi}}{2 \sqrt{\ln 2}} \frac{\Delta \lambda_{s} \Delta \lambda_{0}}{\left(\Delta \lambda_{s}^{2}+\Delta \lambda_{0}^{2}\right)^{1 / 2}} \times \exp \left[-4 \ln 2 \bullet \frac{\left(\lambda_{s 0}-\lambda_{0}\right)^{2}}{\Delta \lambda_{s}^{2}+\Delta \lambda_{0}^{2}}\right] \bullet\left[1-\frac{8 \ln 2\left(\lambda_{s 0}-\lambda_{0}\right)}{\Delta \lambda_{s}^{2}+\Delta \lambda_{0}^{2}} \frac{\varepsilon}{k}\right](5)$

$K_{1}$ is light utilization efficiency, and $A$ is voltage to light power conversion coefficient, $I_{0}$ is the maximum light intensity of laser, $R_{\mathrm{s} 0}$ is PS-FBG reflectivity at center wavelength, $\lambda_{\mathrm{s} 0}$ and $\Delta \lambda_{\mathrm{s}}$ are initial center wavelength and FWHM of $\pi$ PSFBG narrow notch, respectively. $\lambda_{0}$ and $\Delta \lambda_{0}$ are the laser center wavelength and FWHM, separately. $\varepsilon$ is external strain, and $k$ is $\pi$ PS-FBG strain sensitivity coefficient. For the fiber grating around the $1550 \mathrm{~nm}, k=1$ approximately. $\Delta \lambda_{\mathrm{s}}$ is generally $30-50 \mathrm{pm}$ and $R_{\mathrm{s} 0}$ can reach as high as 0.99 as for $\pi \mathrm{PS}-\mathrm{FBG}$, while $\Delta \lambda_{\mathrm{s}}$ and $R_{\mathrm{s} 0}$ of FBG are usually around $0.2-0.6 \mathrm{~nm}$ and 0.9 , respectively. From Formula (5), $\pi$ PS-FBG is more sensitive to ultrasonic wave than FBG. The difference between the two is about one magnitude order. 

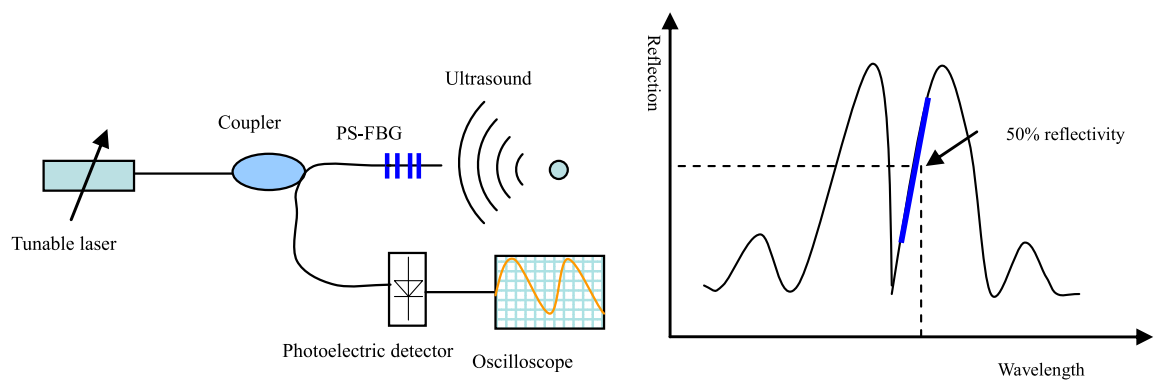

Fig. 1. Edge filter demodulation system

\subsection{Effective detection length}

Fig. 2 shows the reflection spectrum of the $\pi$ PS-FBG under the action of the axial ultrasonic wave with amplitude of $20 \mu \varepsilon$ and the frequency $f=1-10 \mathrm{MHz}$. From Fig. 2, it can be seen that the shape of the PS-FBG reflection spectrum, center wavelength as well as $50 \%$ reflection (the reflectivity at the wavelength whose initial reflectivity is at the middle position of the single slope of the $\pi$ FBG sharp dip, as shown in Fig. 1) remain unchanged when the ultrasonic frequency varies from $1 \mathrm{MHz}$ to $10 \mathrm{MHz}$ and corresponding ultrasonic wave length changes from $6.3 \mathrm{~mm}$ to $0.63 \mathrm{~mm}$, which illustrates that $\pi \mathrm{PS}-\mathrm{FBG}$ can still measure the ultrasonic wave when its grating region length is as much as 16 times of the ultrasonic wave length. Because the ultrasonic wave used for damage detection is generally less than $10 \mathrm{MHz}$ and the ultrasonic wavelength is longer than $1 \mathrm{~mm}$, there is no need to consider the relative magnitude of the grating length to the ultrasonic wave length during conventional damage detection.

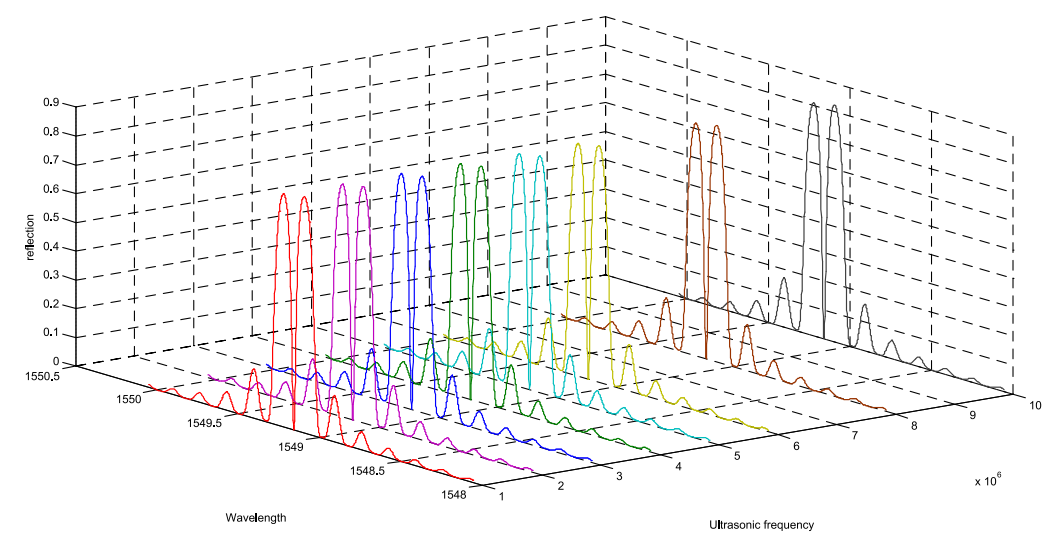

Fig. 2. $\pi$ PS-FBG reflection spectrum under axial ultrasonic wave

\section{Sensing model under transverse ultrasonic wave}

To simplify the analysis, the ultrasonic wave is considered as a pure transverse stress wave $\sigma_{\mathrm{z}}=v\left(\sigma_{\mathrm{x}}+\sigma_{\mathrm{y}}\right)=0$. Assuming ultrasonic waveform change with time according to cosine law, the axis stresses along the $X$ axis and $Y$ axis are:

$$
\sigma_{x}=-\sigma_{y}=P(x, t)=P_{0} \cos \left(\omega t-\frac{2 \pi}{\lambda_{m}} x\right)=P_{0} \cos \left[2 \pi f\left(t-x / v_{m}\right)\right]
$$

When $\pi$ PS-FBG is forced by transverse stress wave, two orthogonal polarization modes namely the fast and slow axes of the fiber are generated. On the basis of the literature [16], the refractive index changes of the two axes are respectively: 


$$
\begin{aligned}
& \Delta n_{x}=-\frac{n_{e f f}^{3}}{2 E}\left\{\left[\left(p_{11}-2 v p_{12}\right) \sigma_{x}\right]+\left[(1-v) p_{12}-v p_{11}\right]\left(\sigma_{y}+\sigma_{z}\right)\right\} \\
& \Delta n_{y}=-\frac{n_{e f f}^{3}}{2 E}\left\{\left[\left(p_{11}-2 v p_{12}\right) \sigma_{y}\right]+\left[(1-v) p_{12}-v p_{11}\right]\left(\sigma_{x}+\sigma_{z}\right)\right\}
\end{aligned}
$$

The center wavelength change of the fast and slow axes:

$$
\begin{aligned}
\Delta \lambda_{x} & =-\frac{n_{e f f}^{3} \Lambda}{E}\left\{\left[\left(p_{11}-2 v p_{12}\right) \sigma_{x}\right]+\left[(1-v) p_{12}-v p_{11}\right]\left(\sigma_{y}+\sigma_{z}\right)\right\}+2 \frac{n_{e f f} \Lambda}{E}\left[\sigma_{z}-v\left(\sigma_{x}+\sigma_{y}\right)\right] \\
\Delta \lambda_{y} & =-\frac{n_{e f f}^{3} \Lambda}{E}\left\{\left[\left(p_{11}-2 v p_{12}\right) \sigma_{y}\right]+\left[(1-v) p_{12}-v p_{11}\right]\left(\sigma_{x}+\sigma_{z}\right)\right\}+2 \frac{n_{e f f} \Lambda}{E}\left[\sigma_{z}-v\left(\sigma_{x}+\sigma_{y}\right)\right]
\end{aligned}
$$

Bringing Formula (6) into the Formula (9) (10), the following equations can be obtained:

$$
\begin{gathered}
\Delta \lambda_{x}=-\Delta \lambda_{y}=-\frac{n_{e f f}^{3} \Lambda}{E}\left[(1+v)\left(p_{11}-p_{12}\right)\right] \sigma_{x} \\
\frac{\Delta \lambda_{x}}{P_{0}}==-\frac{n_{e f f}^{3} \Lambda}{E}\left[(1+v)\left(p_{11}-p_{12}\right)\right]
\end{gathered}
$$

According to the Formula (11) (12), there will be birefringence in $\pi$ PS-FBG under transverse ultrasound, while the center wavelength change along $X$ axis and $Y$ axis are equal and opposite. The center wavelength change sensitivity of $\lambda_{x}$ to $\sigma_{\mathrm{x}}$ is $3.9117 \mathrm{pm} / \mathrm{MPa}$. If the light energy in fiber core is distributed according to that the polarization component amplitude ratio of $X$ axis to $Y$ axis is equal to $1: 1, \pi \mathrm{PS}-\mathrm{FBG}$ reflection spectrum under transverse ultrasonic stress range from $0.1 \mathrm{MPa}$ to 3.2 $\mathrm{MPa}$ are obtained in Fig. 3. From Fig. 3, the transverse ultrasonic wave has no effect on the $\pi$ PS-FBG center wavelength; while the narrow notch is split into two spikes due to birefringence, the difference between the left and right spike is gradually increasing with the ultrasonic intensity, while the center narrow notch is widening and the center wavelength reflectivity is decreasing toward the transverse ultrasonic amplitude. When the transverse force increases to a certain degree (3.2 MPa), there may even be a second division around the depression.

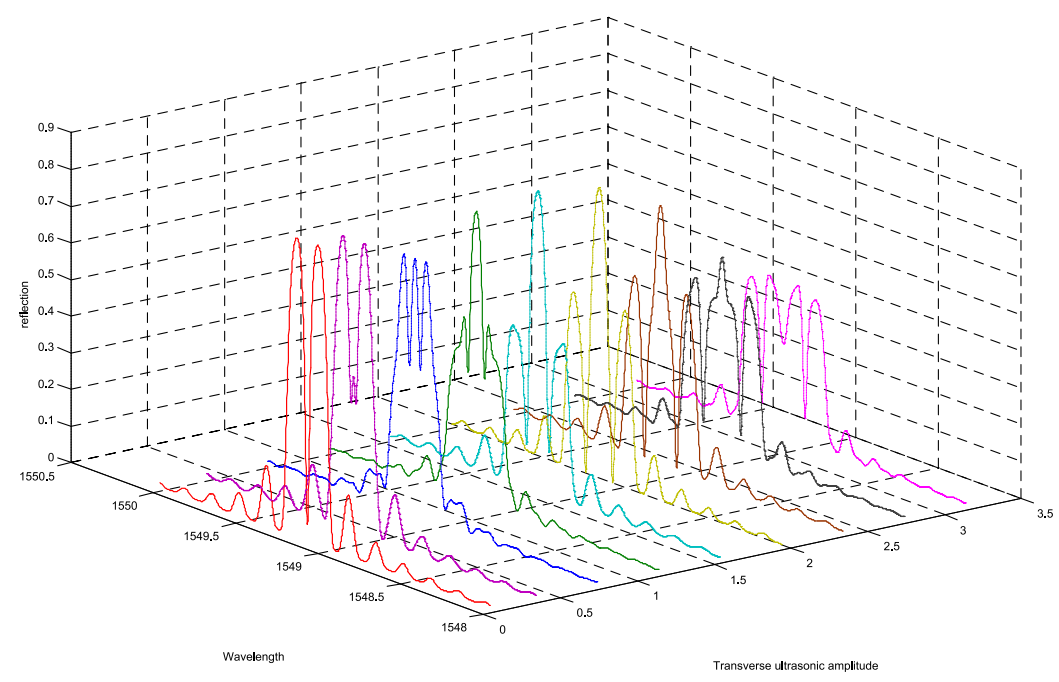

Fig. 3. $\pi \mathrm{PS}-\mathrm{FBG}$ reflection spectrum under transverse ultrasonic wave 
decreases with the transverse ultrasonic amplitude. When the ultrasonic stress changes in $0-1 \mathrm{MPa}$, there is an approximate linear relation between them. The dip FWHM increases gradually with transverse ultrasound. When the transverse stress varies in $0-1.3 \mathrm{MPa}$, the sharp dip FWHM is very small, which can be considered unchanged. When the reflectivity of the dip is higher than that of spikes on both sides, the dip FWHM will change rapidly $(\mathrm{P}=1.4 \mathrm{MPa})$. It may be due to the combine of side lobe and 0 level mode, leading to the nonlinear bandwidth change. There is a linear relationship between the splitting point difference and the transverse ultrasound, and this polarization-related property is consistent with the Feng Kui's experiment result [17].

The laser wavelength is adjusted to the $50 \%$ reflection position of the PS-FBG dip at the initial time in demodulation system based on tunable laser. The system output signal change can be reflected by the reflectivity variation at the setting laser wavelength, which we call the 50\% reflectivity in this paper. From Fig. 4, 50\% reflectivity firstly decreases and then increases with the transverse ultrasonic amplitude. Because of the nonlinear relation, it can not achieve the lateral ultrasound directly through the demodulation system based on tunable laser.
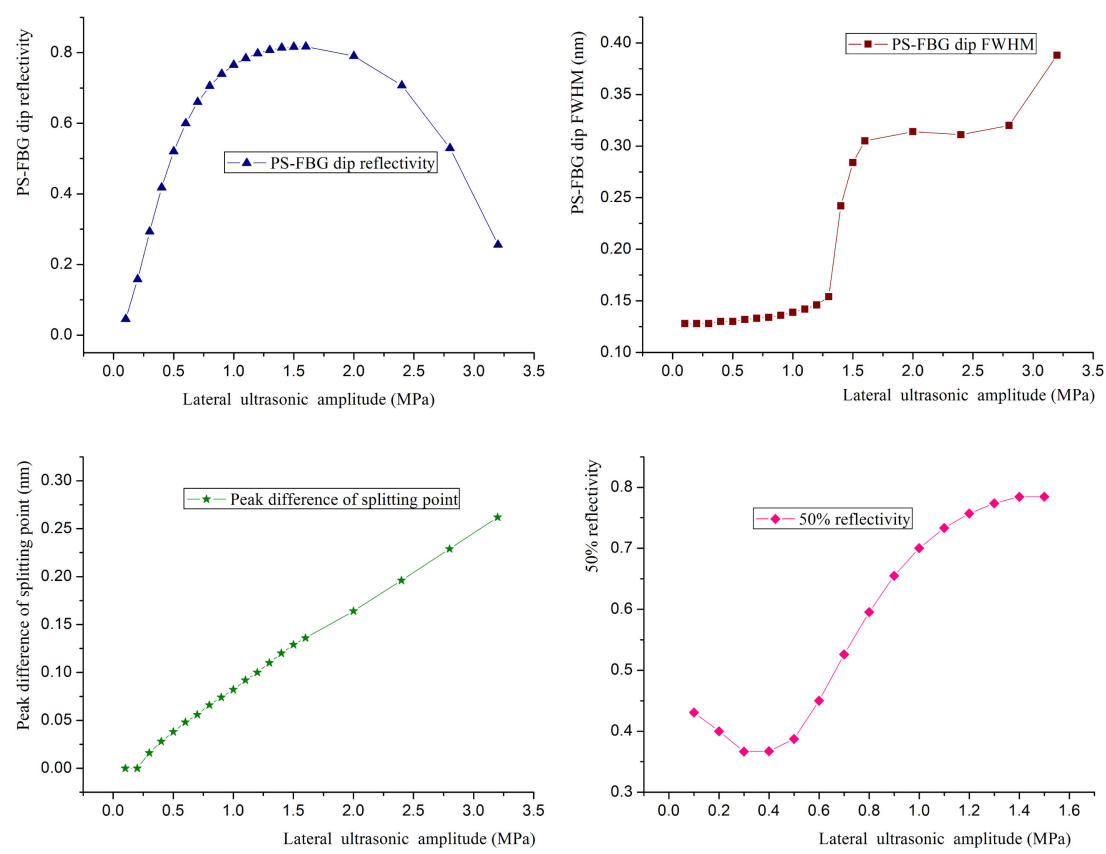

Fig. 4. PS-FBG parameters change with the transverse ultrasonic amplitude

\section{Sensing model under ultrasonic wave from an arbitrarily angle}

PS-FBG reflection spectrum under the ultrasonic stress wave from an arbitrary angle is also analyzed in order to discuss the overall effect of both the axial and transverse force on the reflection spectrum as well as the demodulation signal. The main simulation parameters are as follows: acting join force magnitude $P_{0}=$ $0.72 \mathrm{MPa}$, the material elastic modulus the force acted on $E=7.2 \mathrm{GPa}$, therefore the strain magnitude $A=10 \mathrm{u} \varepsilon$; the incident angle between the force and the grating axis is $\theta$. 


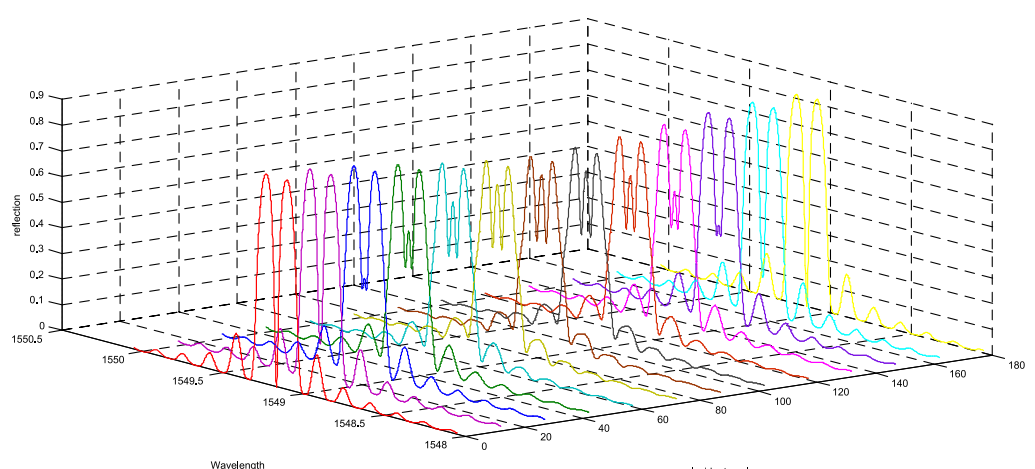

Fig. 5. PS-FBG reflection spectrum at different ultrasonic incident angle
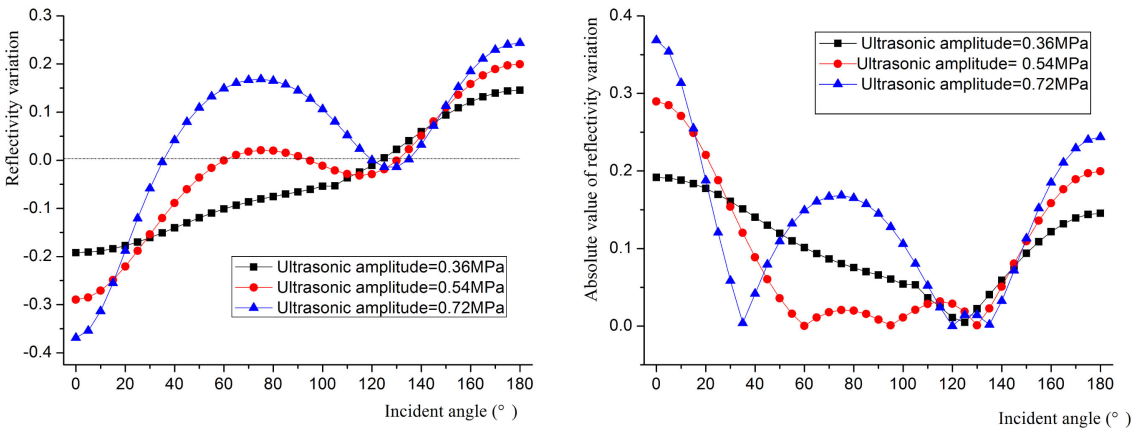

Fig. 6. PS-FBG 50\% reflectivity change with the ultrasonic incident angle

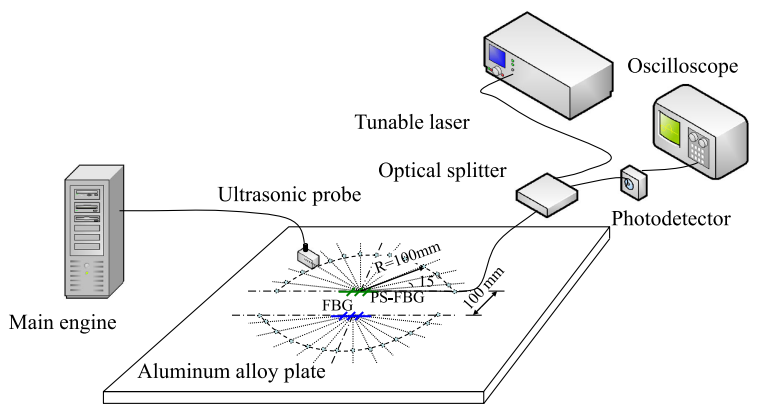

Fig. 7. Experimental principle for PS-FBG 50\% reflectivity change with the ultrasonic incident angle

The variation of PS-FBG reflection spectrum with ultrasonic wave incident angle is shown in Fig. 5. In Fig. 5, under the combined action of axial and transverse forces, the center wavelength and spectral shape are both changed, and the center wavelength increases linearly with the axial component force, while due to the horizontal component force, the narrow notch will split, as the same effect of the equal lateral force alone. According to Fig. 5, the change curve of PSFBG 50\% reflectivity with the ultrasonic incident angle is got in Fig. 6: owing to the influence of the axial and transverse composite forces, the absolute variation of PS-FBG 50\% reflectivity presents Cosine distributions accompanied by two peaks in the middle with the incident angle under different ultrasonic pressure. The two peak shapes in the relation curve depend on the ultrasonic intensity: it appears a small peak and a much bigger one under the ultrasonic amplitude of $0.72 \mathrm{MPa}$, and it has two peaks with nearly the same size under the $0.54 \mathrm{MPa}$ ultrasonic wave, while a peak even disappears under the effect of the $0.36 \mathrm{MPa}$ ultrasonic intensity. It 
is different from the steady Cosine response characteristics of FBG to the twodimensional ultrasonic wave [4]. Because that the relation between output signal and incident angle is not a monotonic function, it is difficult to distinguish the axial and lateral component force from the output signal. As presented in Fig. 6, the relative change of the $50 \%$ reflectivity is not always negative or positive, for example, irradiated by the $0.54 \mathrm{MPa}$ ultrasonic wave, $50 \%$ reflectivity is negative when the ultrasonic incidence angle is in the range of $0^{\circ}-60^{\circ}$. Therefore the PSFBG signal may appear an opposite phase change with different incident angle.

\section{Experimental study}

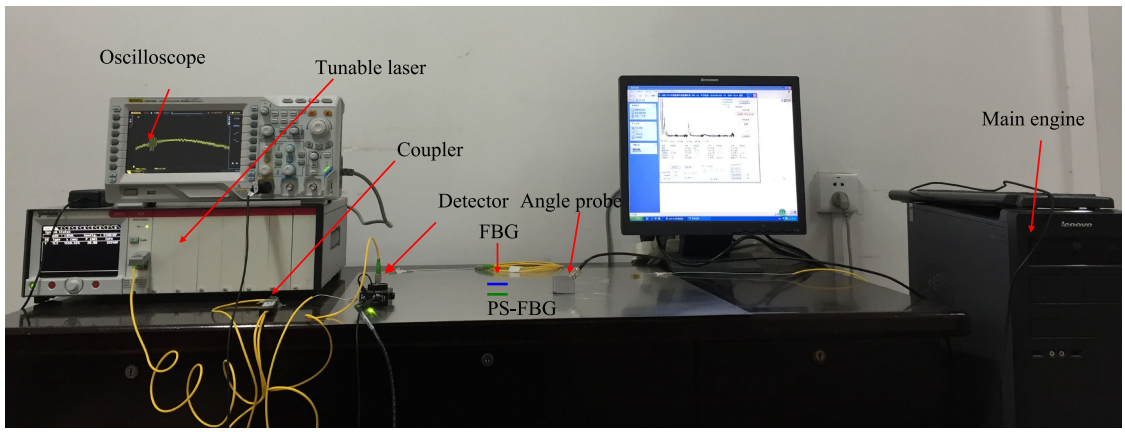

Fig. 8. Arrangement of experimental device

In order to verify the ultrasonic measurement feasibility of the PS-FBG and analyze its sensitivity to the longitudinal and transverse waves, some experimental study was carried out. Fig. 7 and Fig. 8 showed the experimental principle and the layout of the fiber gratings. PS-FBG and FBG were glued parallel on the surface of 5052 aluminum alloy plate $1000 \mathrm{~mm} * 1000 \mathrm{~mm} * 1 \mathrm{~mm}$ in size with $A B$ adhesive, the PS-FBG parameters were as follows: the grating length was $20 \mathrm{~mm}$; the phase shift $\pi$ was located in the middle of the grating region; the initial center wavelength and FWHM of the sharp dip were $1549.872 \mathrm{~nm}$ and $0.032 \mathrm{~nm}$, respectively. The adhesive lengths of the two gratings were both equal to $20 \mathrm{~mm}$. We paid great attention to the adhesive layer size during pasting process to ensure the paste thickness consistency along the grating axis. The ultrasonic excitation devices were consisted of an ultrasonic transmitting and receiving detection card installed in the main engine as well as an ultrasonic angle probe with the center frequency of $1 \mathrm{MHz}$ and incidence angle of $30^{\circ}$. The pulse signal with the voltage amplitude of $200 \mathrm{~V}$ and duration time of 1 us was generated by the detection card to excite the ultrasonic angle probe. Because the ultrasonic frequency thickness product was $1 \mathrm{MHz} \bullet \mathrm{mm}$, there was only the S0 mode of the Lamb wave propagating within the plate according to the dispersion curve of the 5052 aluminum alloy. Both the PSFBG and FBG were demodulated by the system based on tunable laser, and the center wavelength change was obtained according to the optical power received by the optical detector. Yenista-TLS-AG-C tunable laser was used, and its output power and center wavelength were set to $20 \mathrm{~mW}$ and $1549.856 \mathrm{~nm}$, separately. Thorlabs-PDA-10CS was selected as the photoelectric detector in the experiment. The ultrasonic angle probe was moved clockwise along the semicircle around the grating area with a radius of $100 \mathrm{~mm}$ by an uniform spacing. During the moving process, the angle probe was always along the radius direction. The corresponding 
grating measurement results were recorded successively when the ultrasonic probe at each incident position.
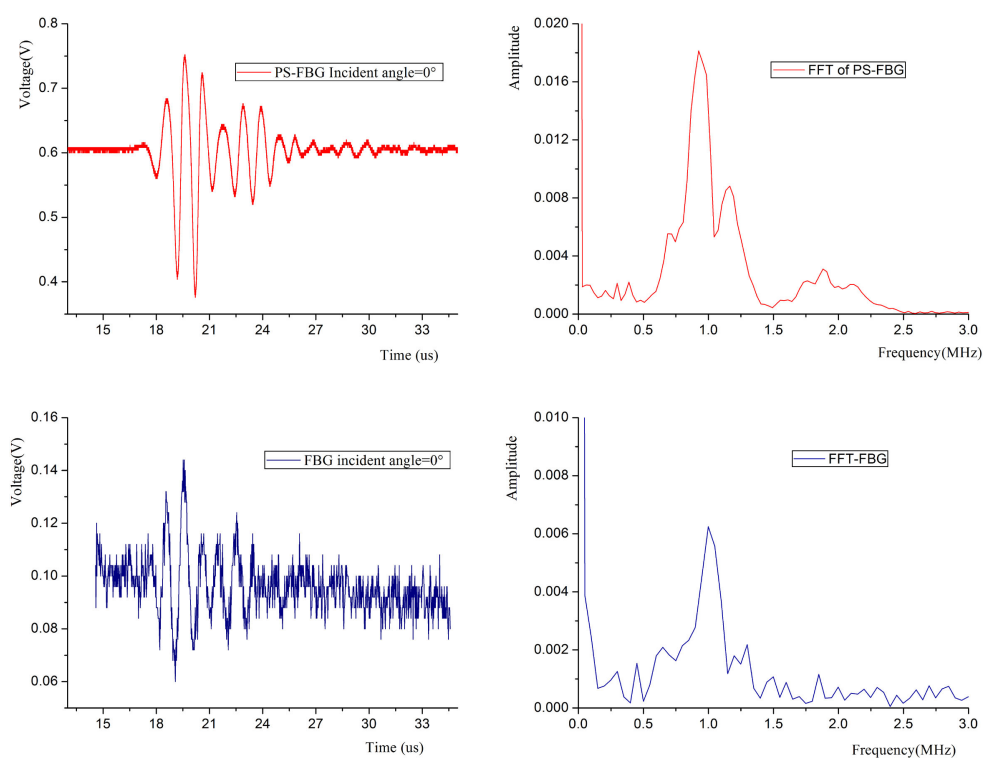

Fig. 9. Signals of PS-FBG and FBG at ultrasonic incident angle $=0^{\circ}$
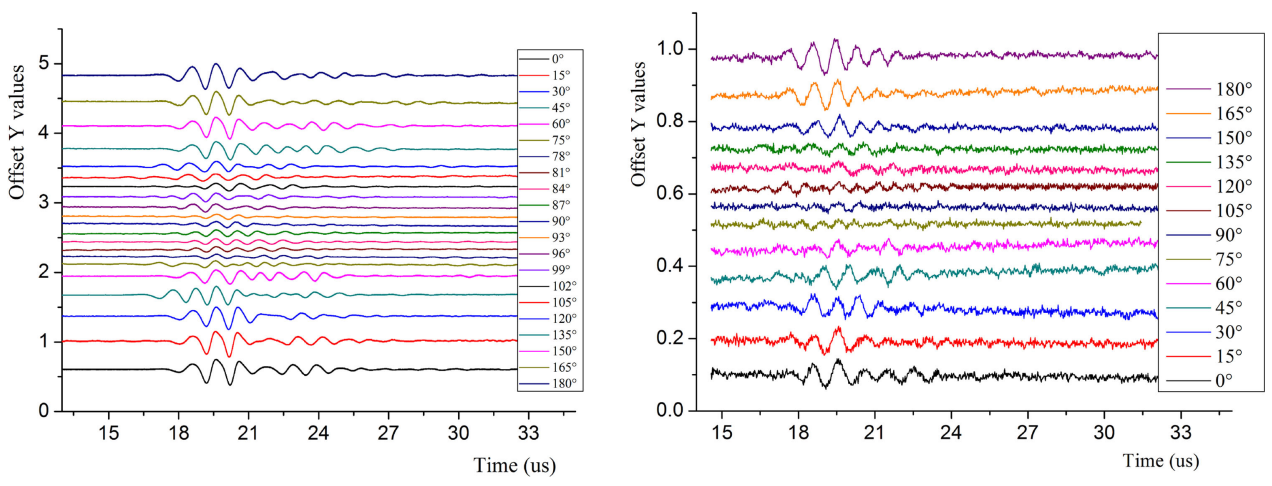

Fig. 10. PS-FBG and FBG test signals at different ultrasonic incident angles

From the measured waveforms in Fig. 9, typical Lamb waves were both measured by PS-FBG and FBG, while PS-FBG had much higher signal strength and signal to noise ratio compared with FBG, which was consistent with the previous analysis. The similar wave shapes were obtained at other measurement position as shown in Fig. 10. In this paper, the first wave packet was considered as the study object to reduce the effect of wave scattering and reflecting, and its peak to peak value was regarded as the evaluation index of ultrasonic intensity, then the relationship between the peak to peak value and the ultrasonic incident angle was obtained in Fig. 11: PS-FBG peak-to-peak value and incident angle showed approximate Cosine relation with two peaks in the middle, which was basically consistent with the previous analysis. The manufacturing error of the grating parameters such as phase shift position, phase-shift value, grating length; the difference of surface bonding structure and layer model as well as the temperature change during experiment may all result in the peak position and amplitude differences between the experiment and simulation result. While, FBG peak-topeak value displayed approximate cosine relation between incident angle. The 
difference distributed response characteristics between PS-FBG and FBG may lie in the more obvious influence of the transverse birefringence on the PS-FBG. As shown in Fig. 10, the PS-FBG signals at incident angle from $0^{\circ}$ to $45^{\circ}$ had opposite phase to the signals from $60^{\circ}$ incident position, which basically agreed with the previous analysis.
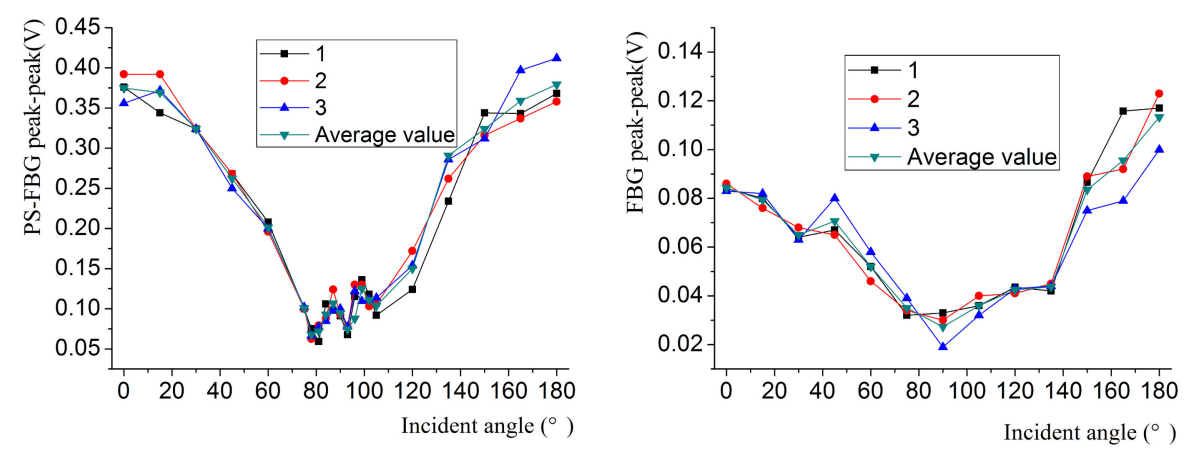

Fig. 11. Variations of peak-to-peak value change with incident angle

In addition, PS-FBG was used to measure the high frequency ultrasound in experiment. A piezoelectric ceramic of $13 \mathrm{~mm} * 13 \mathrm{~mm} * 0.4 \mathrm{~mm}$ in size was droved by the ultrasonic detection card aforementioned with $200 \mathrm{~V}$ impulse voltage and 1 us pulse duration. The ultrasonic wave generated by the piezoelectric ceramics entered the $500 \mathrm{~mm} * 500 \mathrm{~mm} * 0.2 \mathrm{~mm}$ aluminum plate though a plexiglass wedge with an incident angle of $30^{\circ}$. The PS-FBG with grating length of $20 \mathrm{~mm}$ and phase shift $\pi$ in its middle was pasted along the ultrasonic axis $50 \mathrm{~mm}$ far away from the ultrasonic incidence point. The PS-FBG center wavelength was $1550.190 \mathrm{~nm}$, and the demodulation system in Fig. 7 was used to demodulate its wavelength fluctuation. The laser wavelength and power were set as $1550.202 \mathrm{~nm}$ and $20 \mathrm{~mW}$, separately. The PS-FBG ultrasonic signal and its Fourier transform results were displayed in Fig. 12. From Fig. 12, there were mainly two wave packets in time domain, and the main frequency components were $1.40 \mathrm{MHz}$, 3.33 MHz and $12.11 \mathrm{MHz}$ in frequency domain. From the waveform period of the wave packets enlarged view in Fig. 13, the basic frequency of wave packet 1 was 12.11 MHz and that of wave packet 2 were $1.40 \mathrm{MHz}$ and $3.33 \mathrm{MHz}$. According to the aluminum dispersion curve, the group velocities of A0 mode wave at the frequency thickness product $0.280 \mathrm{MHz} \bullet \mathrm{mm}$ and $0.666 \mathrm{MHz} \bullet \mathrm{mm}$, A1 mode wave at the frequency thickness product $2.422 \mathrm{MHz} \bullet \mathrm{mm}$ were $1502 \mathrm{~m} / \mathrm{s}, 2067 \mathrm{~m} / \mathrm{s}$ and $3670 \mathrm{~m} / \mathrm{s}$, respectively. Assuming that the group velocity of the above two A0 hybrid mode was the average of the each A0 mode velocity, $1502 \mathrm{~m} / \mathrm{s}+$ $2067 \mathrm{~m} / \mathrm{s} / 2=1784.5 \mathrm{~m} / \mathrm{s}$, therefore the time difference between the hybrid A0 mode and $2.422 \mathrm{MHzmm} \mathrm{A1} \mathrm{mode} \mathrm{passing} \mathrm{through} 50 \mathrm{~mm}$ distance was $50 \mathrm{~mm} /$ $1784.5 \mathrm{~m} / \mathrm{s}-50 \mathrm{~mm} / 3670 \mathrm{~m} / \mathrm{s}=14.395$ us, which was approximately equal to the 14.7 us time difference of the two wave packet spikes in Fig. 12. Therefore the first wave packet in Fig. 12 was A1 mode with dominant frequency of $12.11 \mathrm{MHz}$, while the second one in Fig. 12 was A0 mode wave packet with dominant frequency of $1.40 \mathrm{MHz}, 3.33 \mathrm{MHz}$. It showed that the PS-FBG $20 \mathrm{~mm}$ in length measured ultrasonic wave up to $12 \mathrm{MHz}$ successfully, which also verified that the response frequency of the PS-FBG to the ultrasound was not limited by its grating length. 

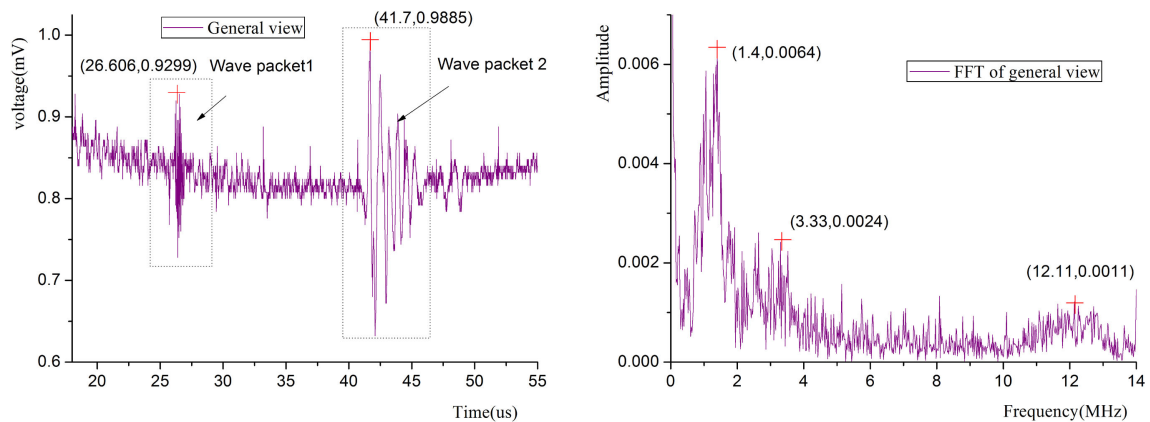

Fig. 12. Ultrasonic signal obtained by PS-FBG from aluminum plate
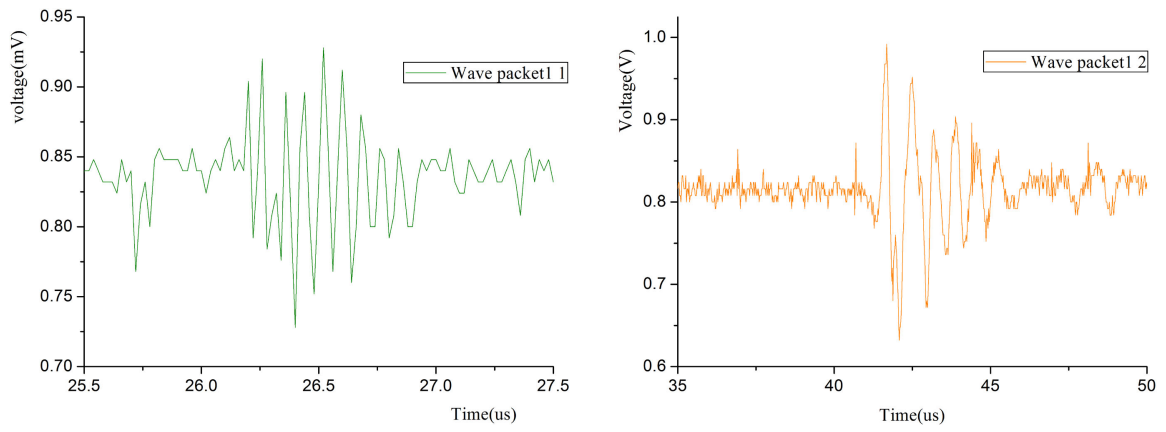

Fig. 13. Enlarged drawing for wave packet

\section{Conclusion}

The PS-FBG sensing models excited by ultrasonic wave from different direction were set up, and the reflection spectrum characteristics of the PS-FBG under different excitation conditions were simulated using the transfer matrix theory. The analysis results were as follows: under the axial ultrasonic excitation, PS-FBG and common FBG had the same center wavelength sensitivity, but PS-FBG had higher signal strength compared with FBG in the demodulation system based on tunable laser, and grating length had little effect on the measurement results; under the action of axial ultrasonic wave, PS-FBG sharp dip would be split due to birefringence, and the distance between the two splitting points was proportional to the transverse ultrasonic intensity, while the peak reflectivity, half bandwidth, 50\% reflectivity would change with transverse ultrasonic wave except the center wavelength of the dip; under the ultrasonic excitation from arbitrary direction, the PSFBG signal intensity and incident angle presented a similar Cosine function relationship with two peaks, and the PS-FBG waveform may have an opposite phase at different excitation positions. Finally, an experimental system based on tunable laser was built to verify the credibility of the simulation analysis and more than $12 \mathrm{MHz}$ ultrasonic wave was detected by PS-FBG.

\section{Acknowledgments}

This paper is supported by National Natural Science Foundation of China under Grant No. 51505187. The authors would like to thank "Wuhan Engineering Research Center of Complex Electromechanical System, Jiang han University" for supporting this work. 